\title{
ABUNDANCES OF REFRACTORY ELEMENTS IN THE ORION NEBULA
}

\author{
R.H. Rubin, E.F. Erickson, M.R. Haas, S.W.J. Colgan, and J.P. Simpson \\ NASA Ames Research Center, M.S. 245 -6, Moffett Field, CA 94035 USA. \\ and R.J. Dufour \\ Dept. Space Physics \&Astronomy, Rice University, Houston, TX 77251 USA.
}

\begin{abstract}
We assess the gas-phase abundances of $\mathrm{Si}, \mathrm{C}$, and Fe from our recent measurements of $\mathrm{Si}^{++}, \mathrm{C}^{++}$, and $\mathrm{Fe}^{++}$in the Orion Nebula by expanding on our earlier "blister" models. The $\mathrm{Fe}^{++} 22.9 \mu \mathrm{m}$ line measured with the $\mathrm{KAO}$ yields $\mathrm{Fe} / \mathrm{H} \sim 3 \times 10^{-6}-$ considerably larger than in the diffuse ISM, where relative to solar, $\mathrm{Fe} / \mathrm{H}$ is down by $\sim 100$. However, in Orion, $\mathrm{Fe} / \mathrm{H}$ is still lower than solar by a factor $\sim 10$. The $\mathrm{C}$ and $\mathrm{Si}$ abundances are derived from new IUE high dispersion spectra of the $\mathrm{C}^{++} 1907,1909 \AA$ and $\mathrm{Si}^{++} 1883,1892 \AA$ lines. Gas-phase $\mathrm{Si} / \mathrm{C}=0.016$ in the Orion ionized volume and is particularly insensitive to uncertainties in extinction and temperature structure. The solar value is 0.098 . Gas-phase $\mathrm{C} / \mathrm{H}=3 \times 10^{-4}$ and $\mathrm{Si} / \mathrm{H}=4.8 \times 10^{-6}$. Compared to solar, $\mathrm{Si}$ is depleted by 0.135 in the ionized region, while $\mathrm{C}$ is essentially undepleted. This suggests that most $\mathrm{Si}$ and $\mathrm{Fe}$ resides in dust grains even in the ionized volume.
\end{abstract}

\section{Model for Orion Nebula}

The model includes a detailed ionization and thermal equilibrium calculation for the ionized gas with an axisymmetric (2-dimensional) geometry. Details and results for the Orion Nebula blister model are in Rubin et al. (1991a = RSHE, 1991b). Here we emphasize the new work on fitting recent International Ultraviolet Explorer Satellite (IUE) and Kuiper Airborne Observatory (KAO) data to determine elemental abundances for $\mathrm{C}, \mathrm{Si}$, and $\mathrm{Fe}$.

\section{Abundances of Refractory Elements}

\subsection{Si/C RATIO}

Silicon and carbon are major constituents of interstellar grains. Their gas-phase abundance ratio may be determined more reliably in nebulae than the ratio of either $\mathrm{C}$ or $\mathrm{Si}$ relative to $\mathrm{H}$. This is the case presently for the ionized volume of Orion. In our model of the Orion Nebula, the dominant ionization states for $\mathrm{C}$ and $\mathrm{Si}$ are $\mathrm{C}^{++}$and $\mathrm{Si}^{++}$with fractional ionizations (RSHE) of 0.59 and 0.79 . The important measurable UV lines from these species - Si III] 1883,92 and C III] $1907,09 \AA$ - arise from energy levels comparably above ground at $\sim 6.57$ and $6.50 \mathrm{eV}$. Therefore the $\mathrm{Si} / \mathrm{C}$ abundance ratio derived from observations of these lines is extremely insensitive to errors in the electron temperature, $T_{e}$, distribution. Also, because the critical densities for these lines are well above the highest density in the model, their volume emissivities have essentially the same dependence on density. Hence, the ratio is insensitive to errors in the density structure. Additionally, because of the proximity of the wavelengths, differential extinction corrections will play a negligible role in the determination of $\mathrm{Si} / \mathrm{C}$. In Figure 1 , we show the best fit of $\mathrm{Si} / \mathrm{C}=0.016$ to our new IUE high-dispersion data. These new observations were made at positions to avoid the bar to the SE (which the model does not address) and greatly expand what had been available. We note that there are virtually parallel arguments for using Si II] $2335-50 \AA$ and $\mathrm{C}_{\text {III }} 2324-29 \AA$ to derive $\mathrm{Si} / \mathrm{C}$. However, according to our model, the fractional ionizations for $\left\langle\mathrm{Si}^{+}\right\rangle$and $\left\langle\mathrm{C}^{+}\right\rangle$are 0.14 and 0.41 . None of these Si II lines are measurable in the IUE spectra. 


\subsection{C/H RATIO}

Important lines for determining $\mathrm{C} / \mathrm{H}$ are $\mathrm{C}$ III] $1907,09 \AA$ and the ratio of their sum to $\mathrm{H} \beta$. We also used the ratio of the $\mathrm{CII}] 2326.1 \AA\left({ }^{4} \mathrm{P}_{5 / 2} \rightarrow{ }^{2} \mathrm{P}_{3 / 2}\right)$ line to $\mathrm{H} \beta$. This line is the strongest of the 5 components that comprise the multiplet. At high dispersion, some of these are resolvable with IUE. Extinction is applied using $\mathrm{C}(\mathrm{H} \beta)$ derived from $\mathrm{H} \alpha$ and $\mathrm{H} \beta$ imagery coincident with the actual IUE field observed and the Orion reddening function $f(\lambda)$. This is from Walter (1991) and is similar in the optical to Torres-Peimbert et al. (1980) and in the UV with Bohlin \& Savage (1981). Nevertheless, the differential extinction correction over such a large wavelength difference as well as the sensitivity to uncertainty in the $T_{e}$ distribution renders the $\mathrm{C} / \mathrm{H}$ abundance ratio more uncertain than $\mathrm{Si} / \mathrm{C}$.

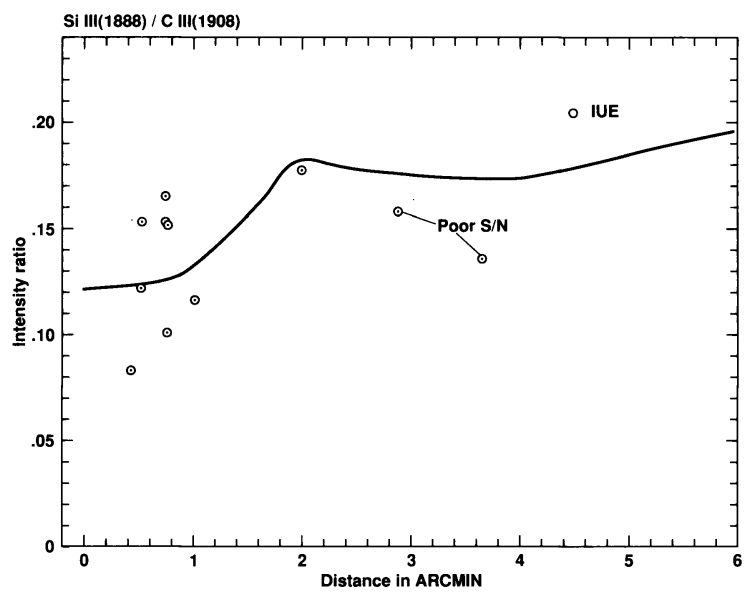

Figure 1. Model predictions for Si $\operatorname{lil}(1883+1892 \AA /$ C III $(1907+1909 \AA)$ intensity ratio vs. projected distance from the center $\left(\theta^{1}\right.$ Ori $\left.\mathrm{C}\right)$. We use the best-fitting model, as described in RSHE with the best fit to our new IUE observations determined for $\mathrm{Si} / \mathrm{C}=0.016$. The data at distances larger than $2.5^{\prime}$ have poor $\mathrm{S} / \mathrm{N}$ and are not used for fitting.

Our $\mathrm{C} / \mathrm{H}$ is determined predominantly on the basis of the $\mathrm{C}$ III data from the dominant state of C. There is a problem with the model in that the typical off-axis electron density, $N_{e}$, values are too high in the singly ionized region (RSHE). Furthermore, the agreement of the $N_{e}$-sensitive ratio C III] $(1907 / 1909)$ with the data is excellent. Tentatively, our best fit obtains for $\mathrm{C} / \mathrm{H}=3 \times 10^{-4}$. This value of $\mathrm{C} / \mathrm{H}$ in the model overestimates $\mathrm{CII}(2326.1) / \mathrm{H} \beta$. This could be explained in a number of ways: a) The model is overestimating $T_{e}$ in the $\mathrm{C}$ II region (RSHE); b) There could be a larger average extinction for the $2326 \AA$ line, which is concentrated farther from the observer than the average for the Balmer lines; c) There could be a lower fractional ionization for $\mathrm{CII}$; d) There could be an actual decrease in the gas-phase $\mathrm{C}$ abundance due to the fact that the $\mathrm{C} I$ region is both closer to the presumed source of grains in OMC 1 and further from the exciting stars. Because a lower $\mathrm{C} / \mathrm{H} \sim 1.7 \times 10^{-4}$ is inferred, this might indicate that the difference is due to less destruction of grains in the $\mathrm{C}^{+}$zone compared with the $\mathrm{C}^{++}$zone.

\section{3. $\mathrm{Si} / \mathrm{H}$ RATIO}

Based on the Si III] $1883,92 \AA$ lines and using the results from Si/C and $\mathrm{C} / \mathrm{H}$ in the above sections, we find $\mathrm{Si} / \mathrm{H}=4.8 \times 10^{-6}$. We repeat that this ratio is not as reliable as $\mathrm{Si} / \mathrm{C}$. This value may be compared with Si/H inferred by Haas et al. (1986) from observations of [Si II] $34.8 \mu \mathrm{m}$ near the Trapezium. When they attribute all of the $34.8 \mu \mathrm{m}$ emission to the photodissociation region (PDR), 
they find $\mathrm{Si} / \mathrm{H}=2.6 \times 10^{-6}$. Using RSHE, and adjusting that calculation for $\mathrm{Si} / \mathrm{H}=4.8 \times 10^{-6}$ in the ionized volume, this model predicts 0.085 of the $34.8 \mu \mathrm{m}$ emission arises in the $\mathrm{H}$ II region. Applying this correction to the PDR abundance, we find $\mathrm{Si} / \mathrm{H}=2.4 \times 10^{-6}$. Taken at face value, the conclusion is that about a factor of 2 enhancement in gas-phase $\mathrm{Si}$ has occurred in the ionized volume. Because the PDR, as the interface with OMC 1, would be the source of fresh grain (and gas) material, this implies some of the dust is destroyed in the harsher environment of the $\mathrm{H}$ II region. Based on a solar Si/H of $3.55 \times 10^{-5}$ (Anders \& Grevesse 1989), the depletions in the PDR and $\mathrm{H}$ II region are 0.068 and 0.135 . Hence, if the total Si abundance were solar in Orion, most of the $\mathrm{Si}$ is locked in grains even in the ionized volume.

\subsection{Fe/H RATIO}

By far the dominant species of iron observable in $\mathrm{H}$ II regions is Fe III. In the present Orion model,

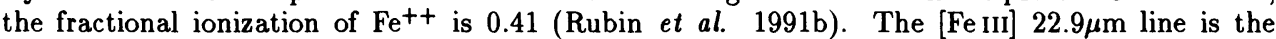
first $\mathrm{Fe}$ line detected in the far-infrared from an $\mathrm{H}$ II region (Erickson et al. 1989). The statistical equilibrium computation for the populations of the lowest 17 energy levels uses the collision strengths of Berrington et al. (1991). Based on recent KAO observations of [Fe III] $22.9 \mu \mathrm{m}$ centered on $\theta^{1}$ Ori $\mathrm{C}$ and the Orion model, we derive $\mathrm{Fe} / \mathrm{H}$. The tentative flux observed with the KAO using the cooled grating spectrometer with full width half maximum beam of $22^{\prime \prime}$, is $\sim 1.5 \times 10^{-18} \mathrm{~W} / \mathrm{cm}^{2}$. The best fit to this line occurs with $\mathrm{Fe} / \mathrm{H} \sim 3 \times 10^{-6}$. We note that the $22.9 \mu \mathrm{m}$ line is predicted to be the brightest $\mathrm{Fe}$ III line in Orion and that the derived $\mathrm{Fe} / \mathrm{H}$ is very insensitive to $T_{\mathrm{e}}$-structure uncertainty or extinction. This ratio may be compared with the solar value. According to Anders \& Grevesse (1989), $\mathrm{Fe} / \mathrm{H}=4.68 \times 10^{-5}$, while Holweger et al. (1990) find a lower solar ratio of $\mathrm{Fe} / \mathrm{H}=$ $3.02 \times 10^{-5}$. Thus in Orion, the depletion of iron is in the range $0.064-0.099$.

In Orion, gas-phase iron is considerably more abundant than in the diffuse ISM. Van Steenberg \& Shull (1988), using Fe II lines in IUE spectra along the line of sight to 12 stars in Ori OB1, find an average depletion for $\mathrm{Fe}$ of 0.0087 . However, in Orion, $\mathrm{Fe} / \mathrm{H}$ is still lower than solar by a factor $\sim 10$ which may indicate most $\mathrm{Fe}$ resides in dust grains even in the ionized volume.

\section{References}

Anders, E., \& Grevesse, N. 1989, Geochim. Cosmochim. Acta, 53, 197.

Berrington, K.A. et al. 1991, J. Phys. B, submitted.

Bohlin, R.C., \& Savage, B.D. 1981, ApJ, 249, 109.

Erickson, E.F., Haas, M.R., Simpson, J.P., Rubin, R.H., \& Colgan, S.W.J. 1989, BAAS, 21, 1156.

Haas, M.R., Hollenbach, D., \& Erickson, E.F. 1986, ApJL, 301, L57.

Holweger, H., Heise, C., \& Kock, M. 1990, Astron. Astrophys., 232, 510.

Rubin, R.H., Simpson, J. P., Hass, M. R., \& Erickson, E. F. 1991a, $A p J, 374,564$ (RSHE).

Rubin, R.H., Simpson, J. P., Haas, M. R., \& Erickson, E. F. $1991 \mathrm{~b}, P A S P, 103,834$.

Torres-Peimbert, S., Peimbert, M., \& Dal tabuit, E. 1980, $A p J, 238,133$.

Van Steenberg, M.E., \& Shull, J.M. 1988, ApJ, 330, 942.

Walter, D.K. 1991, PASP, 103, 830 